\title{
Nonlinear dynamics of a base-isolated beam under turbulent wind flow
}

\author{
Simona Di Nino $(\mathbb{D})$ Angelo Luongo $(\mathbb{D}$
}

Received: 28 November 2020 / Accepted: 25 March 2021 / Published online: 4 April 2021

(C) The Author(s) 2021

\begin{abstract}
A homogeneous continuous viscoelastic beam, describing the dynamics of a base-isolated tower, exposed to a uniformly distributed turbulent wind flow, is studied. The beam is constrained at the bottom end by a nonlinear viscoelastic device, and it is free at the top end. Aeroelastic forces are computed by the quasi-static theory. The steady component of wind is responsible for a Hopf bifurcation, and the turbulent component induces parametric excitation. The interaction between the two bifurcations is investigated. Critical and postcritical behavior is analyzed by perturbation methods. The mechanical performances of the structure are discussed to assess the effectiveness of the viscoelastic isolation system.
\end{abstract}

Keywords Aeroelastic nonlinear behavior - Turbulent wind $\cdot$ Passive control $\cdot$ Perturbation analysis

\section{S. Di Nino}

Department of Civil, Construction-Architectural and Environmental Engineering, University of L'Aquila, 67100 L'Aquila, Italy e-mail: simona.dinino@univaq.it

\section{A. Luongo $(\varangle)$}

International Research Center on Mathematics and Mechanics of Complex Systems, University of L'Aquila, 67100 L'Aquila, Italy

e-mail: angelo.luongo@univaq.it

\section{Introduction}

Slender structures are very sensitive to dynamic actions induced by wind, which causes a variety of instability phenomena [1-6]. The instability and bifurcation events can be related to different kinds of excitation. Structures subjected to steady wind are modeled as self-excited autonomous systems, prone to Hopf bifurcations; structures subjected to turbulent wind are described by parametrically excited non-autonomous systems, therefore potentially suffering divergence, flip or Neimark-Sacker bifurcations. Depending on the nature of the loads, the different kinds of excitation can interact. Some attention has been devoted in literature to various interactive aeroelastic phenomena [711]. Other papers are specifically devoted to analyze galloping versus parametric excitation [12-15], with particular attention to tall buildings [16-21].

In $[12,14]$, the principal resonance of a singledegree-of-freedom system with two-frequency parametric and self-excitation is investigated via the method of multiple scales; qualitative analyses are carried out to determine limit cycles and tori. In [13], analytical investigations of the system under parametric, self-excited and inertial excitation are carried out. In [15] the aeroelastic behavior of a planar prismatic viscoelastic structure, subject to a turbulent wind, is studied. There, a particular class of cross sections is considered, suffering sub-critical bifurcation followed by regain of stability hard loss of stability phenomenon [22-24]). Among the slender structures, the galloping-parametric excita- 
tion of tall buildings is an interesting topic. In [16], the galloping of tall prismatic cantilevered structures, due to unsteady wind, is analytically studied. The structure is subjected to multi-harmonic external and parametric excitation. The multiple-scale method is used to study the effect of primary and secondary resonances on the galloping response of the structure. In $[17,18,21]$ and in $[19,20]$, the parametric, external and self-excitation of one/two towers under turbulent wind flow are investigated, with focus on the periodic and quasi-periodic motions. It is shown that: (i) the unsteady component of wind can cause a significant decrease of the steady critical value; (ii) periodic and quasi-periodic motions exist, according to suitable combinations of the steady and unsteady wind parameters.

To mitigate the dangerous effects of wind, the possibility to add control devices to the structure has been investigated. The aeroelastic control is applied to different kinds of structure, as wind turbines [25,26], bridges [27-30], and, sometimes, tall buildings [31-33]. With reference to these latter, in [33] the aeroelastic behavior of base-isolated tall buildings, exposed to a uniformly distributed steady wind flow, is studied. The passive control is realized by a viscoelastic base isolation system, whose effectiveness is analytically investigated by parametric analyses.

Here, the effect of a turbulent wind flow acting on a tower, of square cross section, is analyzed. A passive control system is introduced, consisting of a viscoelastic device applied to the base of the tower. The interaction between self- and parametrically excited vibrations is studied, both for the uncontrolled and controlled systems. A linear stability analysis is carried out to determine the loci of periodic and quasi-periodic bifurcations. Limit cycles and tori are computed by asymptotic and numerical solutions of the bifurcation equations. The control device is suitably calibrated, aimed to increase the galloping velocity and to reduce the amplitudes of the limit cycle and tori. This paper is an extension of the researches carried out in [15], regarding the interaction between self- and parametric excitation, and developed in [33], concerning the passive control system. The novelties of the current paper consist of: (a) the base isolation is proposed as a passive control system of tall buildings, to mitigate the effects of a turbulent wind, so far developed in [33] against steady wind only; (b) a new class of cross sections is studied, with respect to those analyzed in [15], now manifesting a different scenario triggered by a super- critical bifurcation. By comparing the present analysis with that carried out in the literature on the aeroelastic behavior of (uncontrolled) towers under turbulent wind flow (e.g., [17-21]), other novelties emerge: (a) the MSM is directly applied to PDE, instead of projecting them on a selected mode; (b) the bifurcation scenario is described in a more exhaustive way, by considering the effects of all the bifurcation parameters in a large range.

The paper is organized as follows. In Sect. 2, a baseisolated tower is modeled as a continuous viscoelastic beam under uniformly distributed turbulent wind flow, for which the equations of motion are formulated. In Sect. 3, the bifurcation equations, ruling the slow-flow of the amplitude and phase of the involved mode, are derived via the multiple-scale method. In Sect. 4, the linear stability analysis is carried out, and a three-dimensional stability domain is described. In Sect. 5, a nonlinear analysis of the bifurcation equation is performed, including derivation of the equations ruling the slow-slow-flow and leading to the birth of a torus. In Sect. 6, numerical results are reported for a sample tower, for which an effective base isolation is designed. In Sect. 7, some conclusions are drawn. Two appendixes, supplying details, close the paper.

\section{Aeroelastic model}

The aeroelastic behavior of a tower, of square cross section, dimensions $D \times D$, subjected to a turbulent wind flow of velocity $U(t)$, uniformly distributed along its height, is investigated. A viscoelastic continuous model is formulated. The structural model is assumed to be linear (since, as it is well known, geometric nonlinearities just affect the phase, and not the amplitude, of the response), while the aerodynamic model is nonlinear. An aeroelastic passive control is introduced, by isolating the tower to the base via a nonlinear viscoelastic device.

\subsection{Viscoelastic isolated beam}

The tower is modeled by an equivalent homogeneous beam, consisting of a planar viscoelastic EulerBernoulli beam, obeying to the Kelvin-Voigt constitutive law. The beam is free at the top, and constrained at ground by a viscoelastic isolation system, idealized 

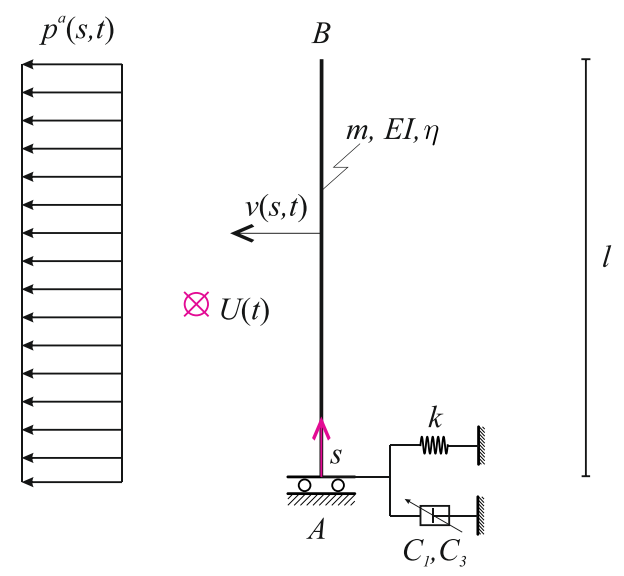

Fig. 1 Base-isolated beam model under transverse uniform turbulent wind flow

as a rheological model made of a linear elastic spring of stiffness $k$ in-parallel with a nonlinear dashpot. This latter is characterized by a linear viscosity coefficient $C_{1}$ and a nonlinear van der Pol-like viscosity coefficient $C_{3}$ (see [33] for details). A model scheme is shown in Fig. 1. The relevant equations of motion and boundary conditions are:

$E I\left(1+\eta \partial_{t}\right) v^{\prime \prime \prime \prime}+m \ddot{v}+c_{e} \dot{v}-p^{a}=0$

$v_{\mathrm{A}}^{\prime}=0$

$E I\left(1+\eta \partial_{\mathrm{t}}\right) v_{\mathrm{A}}^{\prime \prime \prime}+k v_{\mathrm{A}}+C_{1} \dot{v}_{\mathrm{A}}+C_{3} \dot{v}_{\mathrm{A}} v_{\mathrm{A}}^{2}=0$

$E I\left(1+\eta \partial_{\mathrm{t}}\right) v_{\mathrm{B}}^{\prime \prime \prime}=0$

$E I\left(1+\eta \partial_{\mathrm{t}}\right) v_{\mathrm{B}}^{\prime \prime}=0$

Here: $v(s, t)$ is the transverse displacement at the abscissa $s \in(0, l)$ and time $t \in(0, \infty)$, with $l$ the length of the tower; $E I$ is the flexural stiffness of the beam; $m$ is the mass per unit length; $\eta$ is the internal viscous damping coefficient; $c_{\mathrm{e}}$ is the external damping coefficient, accounting for dissipation of the beam in motionless air; $p^{a}(s, t)$ are aerodynamic loads; a dash denotes spatial partial differentiation and $\partial_{t}$ or a dot indicate time partial differentiation. The internal damping is aimed to model internal dissipation via an equivalent viscosity. When it is combined with the external damping, it is able to supply in a consistent (not empirical) basis, the damping factor ratios (see [24]). The internal damping plays an essential role in evaluating the critical wind speed of continuous structures (see [34]).

\subsection{Aerodynamic model}

The aerodynamic forces are determined according to the quasi-steady theory, in which data obtained in wind tunnel static tests are exploited. These experimental tests are related to rigid cylinders, elastically constrained. However, the results are usually considered valid also for flexible cylinders, representative of real structures, by assuming that the aerodynamic forces at the generic abscissa of the cylinder depend exclusively on the speed of the cylinder at that abscissa (i.e., the constitutive law of the forces is assumed to be of local type). In spite this approximation is rather rough and unrealistic, it is usually accepted to formulate analytical models of complex structures $[1,22,35,36]$, in civil as well aeronautical engineering. Moreover, experimental results relevant to towers $[37,38]$ validate the approach.

By assuming the wind velocity is uniform in space, the quasi-static theory [39] supplies the forces as nonlinearly depending on the structural velocity $\dot{v}(s, t)$. Accounting for $\frac{\dot{v}}{U} \ll 1$, and considering the cross section is symmetric with respect to the wind direction, forces are given by the odd-power series:

$p^{a}=-\frac{1}{2} \varrho_{a} D U^{2}\left[A_{1}\left(\frac{\dot{v}}{U}\right)+A_{3}\left(\frac{\dot{v}}{U}\right)^{3}\right]$

where $\varrho_{a}$ is the air mass density and $A_{i}$ are dimensionless aerodynamic coefficients, depending on the cross section shape, of which $D$ is a characteristic length. The aerodynamic forces include all the nonlinearities of the problem. Equation (2) is usually accepted for steady wind flow $U=\bar{U}=$ const; if $U=U(t)$ is a time function, it is still considered valid. This assumption is consistent with the spirit of the quasi-steady theory, which, by considering the fluid-structure system frozen at the generic instant $t$, calls for taking both $\dot{v}=\dot{v}(t)$ and $U=U(t)$ (see, e.g., [16-21]).

By letting:

$U(t)=\bar{U}+u(t)$

in which $\bar{U}$ is the (leading) steady-state wind velocity, and $u(t)$ the (small) turbulent component, and by linearizing Eq. (2) in the ratio $\frac{u}{\bar{U}} \ll 1$, it follows:

$$
\begin{gathered}
p^{a}=-\bar{U}^{2}\left[b_{1}\left(1+\frac{u(t)}{\bar{U}}\right)\left(\frac{\dot{v}}{\bar{U}}\right)\right. \\
\left.+b_{3}\left(1-\frac{u(t)}{\bar{U}}\right)\left(\frac{\dot{v}}{\bar{U}}\right)^{3}\right]
\end{gathered}
$$


where $b_{i}:=\frac{1}{2} \varrho_{a} D A_{i}$ (for $i=1,3$ ) has been introduced to simplify the notation.

In the following, the turbulence is expressed as a combination of harmonics of multiple frequencies, namely:

$u(t)=\sum_{k=1}^{\infty} \hat{u}_{k} \cos k \Omega t$

with $\hat{u}_{k} \ll \bar{U}$ the amplitudes and $k \Omega$ the frequencies of the turbulent component.

\subsection{Non-dimensional equations}

The equations of motion and boundary conditions (1), by accounting for the aerodynamic forces (4) and the turbulence law (5), are recast in the non-dimensional form:

$$
\begin{aligned}
& \left(1+\eta^{*} \partial_{\mathrm{t}}^{*}\right) v^{\prime \prime \prime \prime *}+\ddot{v}^{*}+\left(c_{\mathrm{e}}^{*}+b_{1}^{*} \bar{U}^{*}\right) \dot{v}^{*}+\frac{b_{3}^{*}}{\bar{U}^{*}} \dot{v}^{* 3} \\
& +\left(b_{1}^{*} \dot{v}^{*}-\frac{b_{3}^{*}}{\bar{U}^{* 2}} \dot{v}^{* 3}\right) \sum_{k=1}^{\infty} \hat{u}_{k}^{*} \cos k \Omega^{*} t^{*}=0 \\
& v_{\mathrm{A}}^{\prime *}=0 \\
& \left(1+\eta^{*} \partial_{\mathrm{t}}^{*}\right) v_{\mathrm{A}}^{\prime \prime \prime *}+\kappa v_{\mathrm{A}}^{*}+\zeta_{1} \dot{v}_{\mathrm{A}}^{*}+\zeta_{3} \dot{v}_{\mathrm{A}}^{*} v_{\mathrm{A}}^{* 2}=0 \\
& \left(1+\eta^{*} \partial_{\mathrm{t}}^{*}\right) v_{\mathrm{B}}^{\prime \prime \prime *}=0 \\
& \left(1+\eta^{*} \partial_{\mathrm{t}}^{*}\right) v_{\mathrm{B}}^{\prime \prime *}=0
\end{aligned}
$$

where the following positions have been introduced:

$$
\begin{aligned}
& v^{*}:=\frac{v}{l}, \quad s^{*}:=\frac{s}{l}, \\
& t^{*}:=t \omega_{r}, \quad \Omega^{*}:=\frac{\Omega}{\omega_{r}}, \quad \omega_{r}:=\frac{1}{l^{2}} \sqrt{\frac{E I}{m}} \\
& \eta^{*}=\eta \omega_{r}, \quad c_{e}^{*}=c_{e} \frac{\omega_{r} l^{4}}{E I}, \quad b_{i}^{*}=b_{i} \frac{\omega_{r}^{2} l^{5}}{E I} \\
& \bar{U}^{*}=\frac{\bar{U}}{\omega_{r} l}, \quad \hat{u}_{k}^{*}=\frac{\hat{u}_{k}}{\omega_{r} l} \\
& \kappa:=k \frac{l^{3}}{E I}, \quad \zeta_{1}:=C_{1} \frac{l^{3} \omega_{r}}{E I}, \quad \zeta_{3}:=C_{3} \frac{l^{5} \omega_{r}}{E I}
\end{aligned}
$$

and the star successively dropped. Here, $\omega_{\mathrm{r}}$ is a reference frequency, and dash and dot denote differentiation with respect to the non-dimensional space and time, respectively.

\section{Bifurcation equation}

Attention is focused on the interaction between two different instability mechanisms, potentially exhibited by system (6), namely: (a) galloping (or Hopf bifurcation), which occurs for aerodynamically unstable cross sections $\left(b_{1}<0\right)$ when the steady part $\bar{U}$ of the wind velocity attains a threshold value $\bar{U}_{\mathrm{c}}$, and (b) parametric excitation, which occurs for suitable combinations of the turbulence intensity $\hat{u}$ and frequency $\Omega$. In particular, each component of the turbulence, Eq. (5), parametrically excites the system. However, only some of these, which fall within the unstable areas of Strutt's plane (see, e.g., [40]), are of interest, as the remaining ones generate responses which exponentially decay. Since the higher-order resonance regions become vanishingly small, here the most important case of primary parametric resonance is considered, for which:

$\Omega=2 \omega+\sigma$

$\omega$ being a natural frequency and $\sigma$ a small detuning. Accordingly, the turbulence is represented by its fundamental harmonic alone, assumed to be in primary parametric resonance with the structure, that is $u(t)=\hat{u} \cos ((2 \omega+\sigma) t)$. Therefore, interaction is described in terms of the three bifurcation parameters $\bar{U}, \hat{u}, \sigma$, the first of which is always different from zero, while the remaining ones can vanish.

The multiple-scale method (MSM) is applied (see [41]), to get bifurcation equations (see also [42] for several applications to bifurcation problems). Internal resonances are excluded in this paper and left to future investigations. According to the MSM:

$v(s, t)=A(t) \phi(s) e^{i \omega t}+$ c.c. + h.o.t.

where $\phi(s)$ is an eigenvector of the undamped and unloaded linearized system; $\omega$ is its associated frequency; $A(t)$ is an unknown, slowly variable, complex amplitude; c.c. denotes the complex conjugate of the preceding terms and h.o.t. higher-order terms. The method yields the following complex bifurcation equation (see Appendix A for details):

$$
\begin{aligned}
\dot{A}= & \left(d_{10}+d_{11} \bar{U}\right) A-\frac{d_{11}}{2} \hat{u} e^{i \sigma t} \bar{A} \\
& +\left(d_{30}+\frac{d_{31}}{\bar{U}}\right) A^{2} \bar{A} \\
& +\frac{d_{31}}{2} \frac{\hat{u}}{\bar{U}^{2}} e^{i \sigma t} A \bar{A}^{2}+\frac{d_{31}}{6} \frac{\hat{u}}{\bar{U}^{2}} e^{-i \sigma t} A^{3}
\end{aligned}
$$

where

$$
\begin{aligned}
d_{10}:= & -\frac{c_{\mathrm{e}}}{2}-\frac{1}{I_{\mathrm{m}}}\left(\int_{0}^{1} \eta \phi(s) \phi^{\prime \prime \prime \prime}(s) \mathrm{d} s\right. \\
& \left.+\eta \phi(0) \phi^{\prime \prime \prime}(0)+\zeta_{1} \phi(0)^{2}\right),
\end{aligned}
$$


$d_{11}:=-\frac{b_{1}}{2}$,

$d_{30}:=-\frac{\zeta_{3} \phi(0)^{4}}{I_{\mathrm{m}}}$,

$d_{31}:=-\frac{3 b_{3} \omega^{2}}{I_{\mathrm{m}}} \int_{0}^{1} \phi(s)^{4} \mathrm{~d} s$

with $I_{\mathrm{m}}=\int_{0}^{1} 2 \phi(s)^{2} d s$. Equation (10) generalizes to the turbulent case the equation already derived in [33] for steady wind. The two cases, however, deeply differ each other, since turbulence introduces a stronger coupling between amplitude and phase. The control device enters as follows in the bifurcation equation: (i) the elastic constant $\kappa$ acts on all the $d$ 's coefficients, by the way of the frequency $\omega$ and the shape $\phi(s)$ of the natural mode; (ii) the viscosity coefficients, instead, explicitly appear in the coefficients, namely $\zeta_{1}$ in $d_{10}$ and $\zeta_{3}$ in $d_{30}$. For further analyses, Cartesian and polar real forms of Eq. (10) are derived ahead.

\subsection{Cartesian form}

To recast the complex bifurcation Eq. (10) in real form, $A(t)=Z(t) e^{i \frac{\sigma}{2} t}$ is introduced, with the new variable $Z(t)=X(t)+i Y(t)$ transforming the nonautonomous into autonomous equations. By separating real and imaginary parts, a two-dimensional dynamical system follows:

$$
\left(\begin{array}{c}
\dot{X} \\
\dot{Y}
\end{array}\right)=\boldsymbol{J}_{0}\left(\begin{array}{c}
X \\
Y
\end{array}\right)+\boldsymbol{n}(X, Y)
$$

where

$$
J_{0}:=\left[\begin{array}{cc}
d_{10}+\bar{U} d_{11}-\frac{\hat{u} d_{11}}{2} & \frac{\sigma}{2} \\
-\frac{\sigma}{2} & d_{10}+\bar{U} d_{11}+\frac{\hat{u} d_{11}}{2}
\end{array}\right]
$$

is the Jacobian matrix at the origin, and $\boldsymbol{n}=\left(n_{x}, n_{y}\right)^{T}$ the nonlinear terms vector, of components:

$$
\begin{aligned}
& n_{x}=\left(d_{30}+\frac{d_{31}}{\bar{U}}\right) X Y^{2}+X^{3}\left(d_{30}+\frac{d_{31}}{\bar{U}}+\frac{2 d_{31} \hat{u}}{3 \bar{U}^{2}}\right) \\
& n_{y}=\left(d_{30}+\frac{d_{31}}{\bar{U}}\right) X^{2} Y+Y^{3}\left(d_{30}+\frac{d_{31}}{\bar{U}}-\frac{2 d_{31} \hat{u}}{3 \bar{U}^{2}}\right)
\end{aligned}
$$

In the new variables, the leading part of motion law (9) reads:

$v(s, t)=2\left[X(t) \cos \left(\frac{\Omega}{2} t\right)-Y(t) \sin \left(\frac{\Omega}{2} t\right)\right] \phi(s)$

where Eq. (8) has been used.

\subsection{Polar form}

To recast the complex Eq. (10) in polar form, $A(t)=$ $\frac{1}{2} a(t) e^{i \varphi(t)}$ is posed, which leads to two real equations:

$$
\begin{aligned}
\dot{a}= & \left(d_{10}+d_{11} \bar{U}-\frac{1}{2} d_{11} \hat{u} \cos \gamma\right) a \\
& +\left(\frac{d_{30}}{4}+\frac{d_{31}}{4 \bar{U}}+\frac{d_{31}}{6} \frac{\hat{u}}{\bar{U}^{2}} \cos \gamma\right) a^{3} \\
a \dot{\gamma}= & \left(d_{11} \hat{u} \sin \gamma-\sigma\right) a \\
& -\frac{d_{31}}{6} \frac{\hat{u}}{\bar{U}^{2}} \sin \gamma a^{3}
\end{aligned}
$$

where the phase difference $\gamma:=2 \varphi(t)-\sigma t$ has been introduced. The law of motion (9), at the leading order, becomes:

$v(s, t)=a(t) \phi(s) \cos \left(\frac{\Omega}{2} t+\frac{\gamma(t)}{2}\right)$

The two representations are related by $a=2 \sqrt{X^{2}+Y^{2}}$ and $\gamma=2 \arg (X+i Y)$.

\section{Linear stability analysis}

Stability of the equilibrium position $A=0$ is studied by the Cartesian form (12). It is governed by the eigenvalues of the Jacobian matrix $\boldsymbol{J}_{0}$ :

$\lambda_{ \pm}=d_{10}+d_{11} \bar{U} \pm \frac{1}{2} \sqrt{\triangle(\hat{u}, \sigma)}$

where:

$\Delta(\hat{u}, \sigma):=d_{11}^{2} \hat{u}^{2}-\sigma^{2}$

Right and left eigenvectors, solving $\left(\boldsymbol{J}_{0}-\lambda_{ \pm} \boldsymbol{I}\right) \boldsymbol{x}=\mathbf{0}$ and $\left(\boldsymbol{J}_{0}-\bar{\lambda}_{ \pm} \boldsymbol{I}\right) \boldsymbol{y}=\mathbf{0}$, respectively, are:

$\boldsymbol{x}_{ \pm}=\left(\begin{array}{c}d_{11} \hat{u} \mp \sqrt{\triangle(\hat{u}, \sigma)} \\ \sigma\end{array}\right)$,

$\boldsymbol{y}_{ \pm}=\left(\begin{array}{c}-d_{11} \hat{u} \pm \sqrt{\Delta(\hat{u}, \sigma)} \\ \sigma\end{array}\right)$

When turbulence is absent $(\hat{u}=0, \sigma=0), \lambda_{ \pm}$are real and coincident. When $\lambda_{+}=\lambda_{-}=0$, a Hopf bifurcation occurs, at the critical galloping velocity $\bar{U}_{\mathrm{c}}:=-\frac{d_{10}}{d_{11}}$.

The turbulent component modifies the bifurcation conditions, which must be described in a threedimensional $(\sigma, \hat{u}, \bar{U})$ parameter space, in which $\bar{U}$ is the distinguished parameter, and, $\hat{u}$ and $\sigma$ are the 
splitting parameters. The linear stability diagram will be shown later in discussing the numerical results (see Fig. 2). There, flip bifurcations and Neimark-Sacker bifurcations occur, respectively, on the conic and the planar branches of the domain, according to the following discussion.

- When $\Delta(\hat{u}, \sigma)>0$, the eigenvalues are real and distinct. When $\bar{U}$ is such that $\lambda_{ \pm}=0$ (defining a cone in the parameter space), two successive flip bifurcations occur, at which a periodic response, of period double of that of excitation, is triggered. The lowest bifurcation $\left(\lambda_{+}=0\right)$ manifests at a steady wind velocity $\bar{U}(\hat{u}, \sigma)<\bar{U}_{\mathrm{c}}$, so that turbulence reduces the galloping velocity.

- When $\Delta(\hat{u}, \sigma)<0$, the eigenvalues are complex conjugates. Here, when $\bar{U}$ is such that $\operatorname{Re}\left[\lambda_{+}\right]=$ $\operatorname{Re}\left[\lambda_{-}\right]=0$, a Neimark-Sacker bifurcation occurs, at which a quasi-periodic response is triggered. This happens at $\bar{U}=\bar{U}_{\mathrm{c}} \forall(\hat{u}, \sigma)$. The periodic motion experienced by the system under nonturbulent wind is modified by the turbulence, which introduces a second frequency, $\operatorname{Im}\left[\lambda_{ \pm}\right]$, into the response, thus transforming the limit cycle in a torus.

- When $\triangle(\hat{u}, \sigma)=0$, the eigenvalues are real and coincident. They vanish at $\bar{U}=\bar{U}_{\mathrm{c}}$, where the Neimark-Sacker bifurcation degenerates in a flip bifurcation. The splitting parameters decide about the coalescence.

It is worth noticing that, referring to the slow-flow ruled by the bifurcation Eq. (10), the flip bifurcation appears as a divergence (simple zero eigenvalue) and the Neimark-Sacker as a Hopf bifurcation.

Concerning the base isolation, it produces a shift of the eigenvalues Eqs. (18) via the coefficient $d_{10}$, depending of the elastic spring $\kappa$ and of the linear dashpot $\zeta_{1}$. This entails a translation along the $\bar{U}$-axis of the bifurcation locus. Therefore, the linear part of the control device, whose parameters have to be opportunely calibrated, can enlarge the stability domain.

\section{Nonlinear analysis}

A nonlinear bifurcation analysis is carried out, aimed at determining limit cycles (periodic solutions) and tori (quasi-periodic solutions). The former are generated by flip bifurcations, and the latter by the Neimark-Sacker bifurcations.

\subsection{Limit cycles}

By letting $a=$ const, $\gamma=$ const in Eqs. (16) and eliminating the circular functions, the following equation is drawn:

$$
\begin{gathered}
\frac{9 \bar{U}^{2}\left(a^{2} d_{30} \bar{U}+a^{2} d_{31}+4 d_{10} \bar{U}+4 d_{11} \bar{U}^{2}\right)^{2}}{4 \hat{u}^{2}\left(3 d_{11} \bar{U}^{2}-a^{2} d_{31}\right)^{2}} \\
+\frac{36 \sigma^{2} \bar{U}^{4}}{\hat{u}^{2}\left(6 d_{11} \bar{U}^{2}-a^{2} d_{31}\right)^{2}}=1
\end{gathered}
$$

whose solution calls for numerical methods. However, a closed-form solution is obtained at the perfect resonance $(\sigma=0)$ :

$$
\begin{aligned}
& a_{1 \pm}= \pm \frac{\sqrt{6} \bar{U} \sqrt{-2 d_{10}-2 d_{11} \bar{U}+d_{11} \hat{u}}}{\sqrt{3 d_{30} \bar{U}^{2}+3 d_{31} \bar{U}+2 d_{31} \hat{u}}}, \quad \gamma_{1}=0 \\
& a_{2 \pm}= \pm \frac{\sqrt{6} \bar{U} \sqrt{-2 d_{10}-2 d_{11} \bar{U}-d_{11} \hat{u}}}{\sqrt{3 d_{30} \bar{U}^{2}+3 d_{31} \bar{U}-2 d_{31} \hat{u}}}, \quad \gamma_{2}=\pi
\end{aligned}
$$

These are multi-valued functions, whose domain of existence depends by the bifurcation parameters and aerodynamics coefficients.

Stability of the periodic solution $\left(a_{e}, \gamma_{e}\right)$ is governed by the variational equation:

$$
\left(\begin{array}{l}
\delta \dot{a} \\
\delta \dot{\gamma}
\end{array}\right)=J_{e}\left(\begin{array}{l}
\delta a \\
\delta \gamma
\end{array}\right)
$$

where $J_{\mathrm{e}}:=\left[J_{i j}\left(a_{e}, \gamma_{e}\right)\right]$ is the $2 \times 2$ Jacobian matrix at the equilibrium, whose coefficients are:

$$
\begin{aligned}
J_{11}\left(a_{\mathrm{e}}, \gamma_{\mathrm{e}}\right)= & d_{10}+\bar{U} d_{11} \\
& +\left(d_{30}+\frac{d_{31}}{\bar{U}}\right) \frac{3 a_{\mathrm{e}}^{2}}{4} \\
& +\left(-\frac{1}{2} \hat{u} d_{11}+\frac{\hat{u} d_{31} a_{\mathrm{e}}^{2}}{2 \bar{U}^{2}}\right) \cos \gamma_{\mathrm{e}} \\
J_{12}\left(a_{\mathrm{e}}, \gamma_{\mathrm{e}}\right)= & \left(\frac{1}{2} \hat{u} d_{11} a_{\mathrm{e}}-\frac{\hat{u} d_{31} a_{e}^{3}}{6 \bar{U}^{2}}\right) \sin \gamma_{e} \\
J_{21}\left(a_{\mathrm{e}}, \gamma_{\mathrm{e}}\right)= & -\frac{\hat{u} d_{31} a_{\mathrm{e}}}{3 \bar{U}^{2}} \sin \gamma_{\mathrm{e}} \\
J_{22}\left(a_{\mathrm{e}}, \gamma_{\mathrm{e}}\right)= & \left(\hat{u} d_{11}-\frac{\hat{u} d_{31} a_{\mathrm{e}}^{2}}{6 \bar{U}^{2}}\right) \cos \gamma_{\mathrm{e}}
\end{aligned}
$$

The periodic solution is stable if the real part of both the eigenvalues of $\boldsymbol{J}_{\mathrm{e}}$ is negative.

\subsection{Tori}

To analytically investigate tori, the MSM is applied again to the Cartesian bifurcation Eq. (12), to evalu- 
ate the branches of limit cycles emanating from the Neimark-Sacker points. Here, the Jacobian matrix $\mathbf{J}_{0}$ admits purely imaginary eigenvalues $\lambda_{ \pm}= \pm i \varpi$, with $\varpi:=\frac{1}{2} \sqrt{-\Delta}$, while $\bar{U}=\bar{U}_{c}$. Close to these points:

$$
\left(\begin{array}{l}
X \\
Y
\end{array}\right)=R(t)\left(\begin{array}{c}
d_{11} \hat{u}-2 i \varpi \\
\sigma
\end{array}\right) e^{i \varpi t}+\text { c.c. }+ \text { h.o.t. }
$$

where $R(t)=\frac{1}{2} r(t) e^{i \theta(t)}$ is a complex amplitude, and where use has been made of Eq. (20). By letting $\bar{U}=$ $\bar{U}_{c}+\epsilon \Delta U$, the MSM yields the bifurcation equation that rules the slow-slow-motion (see Appendix B for details):

$$
\dot{R}=d_{11} \Delta U R+\left(c_{30 r}+c_{31 r}+i c_{31 i} \hat{u}^{2}\right) \sigma^{2} R^{2} \bar{R}
$$

where

$$
c_{30 r}:=4 d_{30}, \quad c_{31 r}:=\frac{4 d_{31}}{\bar{U}_{c}}, \quad c_{31 i}:=\frac{d_{31} d_{11}}{\bar{U}_{c}^{2} \varpi}
$$

are real coefficients. In real form, Eq. (26) reads:

$$
\begin{aligned}
& \dot{r}=r d_{11} \Delta U+\left(\frac{c_{30 r}+c_{31 r}}{4}\right) \sigma^{2} r^{3} \\
& r \dot{\theta}=\frac{c_{31 i}}{4} \sigma^{2} \hat{u}^{2} r^{3}
\end{aligned}
$$

whose fixed points $r=$ const are:

$$
\begin{aligned}
& r=\frac{2}{\sigma} \sqrt{-\frac{d_{11} \Delta U}{\left(c_{30 r}+c_{31 r}\right)}} \\
& \theta(t)=\beta t+\theta_{0}
\end{aligned}
$$

in which $\theta_{0}$ is an initial phase and $\beta:=\left(\frac{c_{31 i}}{4} \sigma^{2} \hat{u}^{2}\right) r^{2}$ is a frequency correction.

Stability is governed by the variation equation:

$\delta \dot{r}=\left(d_{11} \Delta U+\frac{3}{4}\left(c_{30 r}+c_{31 r}\right) \sigma^{2} r^{2}\right) \delta r$

According to Eqs. (9), $A(t)=(X(t)+i Y(t)) e^{i \frac{\sigma}{2} t}$ and Eq. (25), the quasi-periodic motion, at the leading order, is:

$$
\begin{gathered}
v(s, t)=2 r \sigma\left(\cos \left(\frac{\Omega}{2} t\right) \cos \left(\varpi_{\beta} t+\theta_{0}+\alpha\right)\right. \\
\left.-\sin \left(\frac{\Omega}{2} t\right) \cos \left(\varpi_{\beta} t+\theta_{0}\right)\right) \phi(s)
\end{gathered}
$$

in which two frequencies exist, the driving one, $\frac{\Omega}{2}$, and the amplitude-dependent modulating one, $\varpi_{\beta}:=$ $\varpi+\beta$. Moreover, $\alpha:=\arctan \left(\frac{2 \varpi}{\sigma_{M}(\hat{u})}\right)$, with $\sigma_{M}:=$ $d_{11} \hat{u}$. The modulating amplitude:

$a(t)=\left(2 r \sigma \sqrt{1-\frac{\sigma_{M}(\hat{u})}{\sigma} \cos \left(2 \varpi_{\beta} t+2 \theta_{0}+\alpha\right)}\right)$

exists only when $\sigma>\sigma_{M}(\hat{u})$ (minimum detuning); it spans the range:

$a(t) \in\left[2 r \sigma \sqrt{1-\frac{\sigma_{M}(\hat{u})}{\sigma}}, 2 r \sigma \sqrt{1+\frac{\sigma_{M}(\hat{u})}{\sigma}}\right]$

whose end values are the lengths of the axes of an elliptical trajectory traveled in the $(X, Y)$ state space (see Fig. 10). Note that the ratio between the maximum and minimum amplitudes is predicted to be independent of the steady wind velocity; this aspect will be commented ahead.

\section{Numerical results}

The (uncontrolled) sample system consists of a supertall tower, inspired by [43], having height $l=300 \mathrm{~m}$ and square cross section, $b=12 \mathrm{~m}$ wide. The total stiffness is $E I=2.95 \times 10^{13} \mathrm{Nm}^{2}$, mass density $m=45000 \mathrm{~kg} / \mathrm{m}$, damping parameters $\eta=0.00091 \mathrm{~s}$, and $c_{\mathrm{e}}=320 \mathrm{Ns} / \mathrm{m}^{2}$ (corresponding to the damping ratio $\xi=0.4 \%$ in the first mode). The air mass density is $\varrho=1.25 \mathrm{~kg} / \mathrm{m}^{3}$ and the dimensionless aerodynamic coefficients for the squared cross section are $A_{1}=-0.9298, A_{3}=7.677$ (provided by $[1,22]$ and confirmed via wind tunnel tests in $[16,44]$ ). This example is representative of a class of towers, whose non-dimensional parameters are: $\eta^{*}=0.00026$, $c_{\mathrm{e}}^{*}=0.025$ and $b_{1}^{*}=-0.04649, b_{3}^{*}=0.38385$. The numerical values are consistent with the ordering performed in the perturbation analyses. The parameters of the control system are opportunely designed below.

\subsection{Linear analysis}

The linear stability domain is shown in Fig. 2, by (a) a 3D plot and (b-d) planar contour plots, for both uncontrolled (U) and linearly controlled (LC) systems. Plots are extended beyond the limits of validity of the theory, 
Fig. 2 Linear instability domain: a 3D plots of the $\mathrm{U}$ (orange) and LC (yellow) systems; contour plots of the $\mathrm{U}$ (gray) and LC (black) systems in the planes: $\mathbf{b}$ $(\hat{u}, \bar{U})$ when $\sigma=0.005$; c $(\sigma, \bar{U})$ when $\hat{u}=0.2 ;(\mathbf{d})$ $(\sigma, \hat{u})$ when $\bar{U}=0.49$. The isolation device parameters are $\kappa=8$ and $\zeta_{1}=0.02$

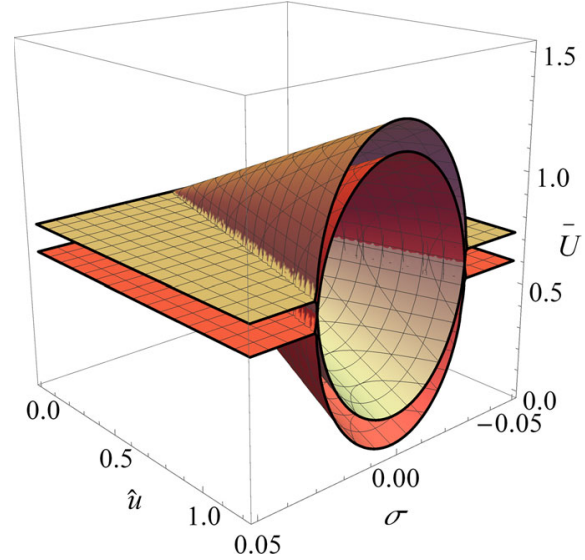

(a)

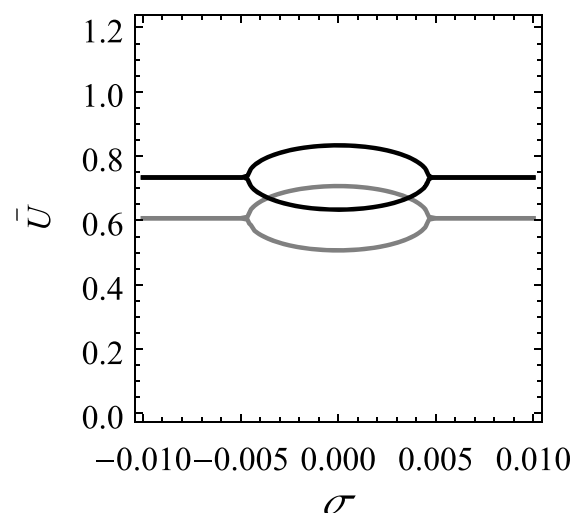

(c)

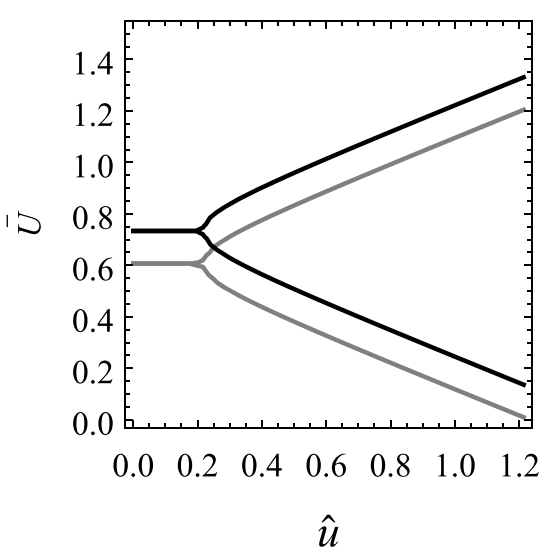

(b)

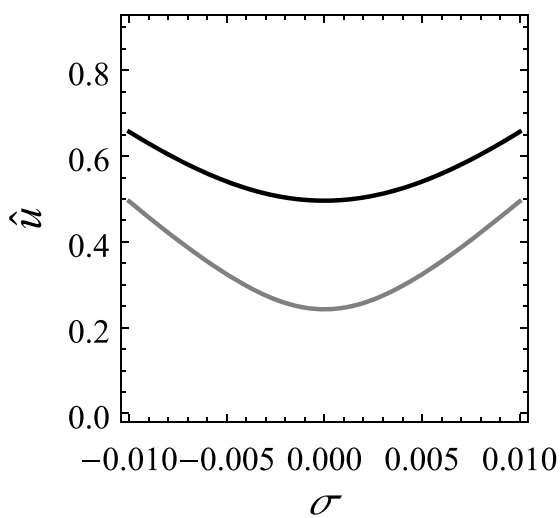

(d) which requires $\hat{u} \ll \bar{U}$. It is observed that the critical wind velocity decreases when the turbulence increases and/or the detuning decreases. The isolation devices are calibrated in order to produce an upward (beneficial) translation of the domain, according the procedure illustrated ahead.

By referring to the uncontrolled system, the (nondimensional) first natural frequency and the corresponding galloping velocity, in the absence of turbulence, are, respectively, $\omega=3.5$ (i.e., $1 \mathrm{rad} / \mathrm{s}$ in dimensional form) and $\bar{U}_{\mathrm{c}}=0.61$ (i.e., $51 \mathrm{~m} / \mathrm{s}$ in dimensional form). More dangerous instability conditions occur when the turbulent wind component is considered. For example, a turbulent wind having amplitude $\hat{u}=0.25$ and detuning $\sigma=0$ (resonant case) reduces the galloping velocity of $20 \%(\bar{U}=0.48)$. Under these wind conditions, the effect of the control system on the critical velocity is investigated. The influence of the linear damping $\zeta_{1}$ is shown in Fig. 3 for a set of selected spring stiffness $\kappa$. It is seen that the purely elastic base isolation produces a detrimental lowering of the critical wind velocity, making essential to add a dashpot. Although this is more efficient when the spring stiffness is low, very soft springs can cause undesired large base displacements. By taking into account these aspects and in order to increase the critical velocity by 1.25 -times that of the uncontrolled system (denoted in figure as $\bar{U}_{u}$ ), the linear isolation parameters $\kappa=8$ and $\zeta_{1}=0.02$ are identified. As a consequence, the non-dimensional frequency of the first natural mode becomes $\omega=2.3$, and the galloping velocity is $\bar{U}=0.61$.

\subsection{Limit cycle analysis}

The nonlinear periodic motions of the system are analyzed at the perfect resonance $(\sigma=0)$ and in a quasiresonant case $(\sigma=0.005)$. The relevant bifurcation 


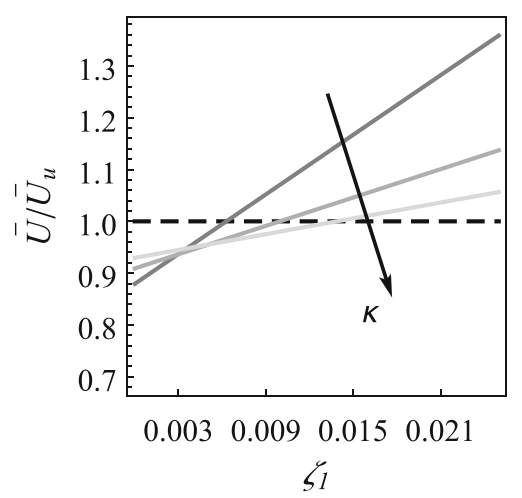

Fig. 3 Critical wind velocity $v s$ the linear viscosity coefficient $\zeta_{1}$, for assigned values of spring stiffness $\kappa=8,16,24$. Black dashed line denotes the critical wind velocity of the uncontrolled system. Turbulence parameters are $\hat{u}=0.25$ and $\sigma=0$

analysis calls for determining limit cycles and analyzing their stability. The influence of the nonlinear viscosity coefficient $\zeta_{3}$ in the nonlinearly controlled (NLC) system is also investigated.

The existence domains of each solution branch (of Eq. (21)) in the parameter plane is shown in Fig. 4 for the LC system $\left(\zeta_{3}=0\right)$. In the same figures, the bifurcation loci are marked in black. The plot displays the existence of one (light gray regions) or two (coexisting) limit cycles (dark gray regions).

Bifurcation diagrams $(a, \bar{U})$ are built up and shown ahead, both in the resonant (Fig. 5) and quasi-resonant (Fig. 6) cases, for selected values of $\hat{u}$. These latter (denoted by the ticks in Fig. 4a for the resonant case and in Fig. $4 \mathrm{~b}$ for the quasi-resonant case) are chosen with the aim to study all the possible bifurcation scenarios. Accordingly, plots are extended beyond the limits of validity of the theory, which requires both a small turbulent component and small motion amplitudes. A comparison between U (gray lines), LC (black lines) and NLC (red lines) systems is made.

The perfect resonant case is studied first (Fig. 5). Bifurcation diagrams are shown there, in which the amplitude of the limit cycles, as defined in Eq. (22), is reported for nonlinear viscosity coefficient $\zeta_{3}=50$. Concerning the LC system, it is observed what follows. The path I (in Fig. 5a) is characterized by three families of limit cycles, all branching from the trivial path; two of them (one stable and the other unstable) are generated when $\bar{U}$ is increased, while a third (unstable) family bifurcates from $\bar{U}=0$ (at which, however, the theory loses validity). By increasing the turbulence amplitude, the two lowest curves come closer in path II (see Fig. 5b), until they merge in path III (see Fig. 5c). Here, once the trivial path loses stability, the system jumps to a higher-amplitude limit cycle. The dangerous effect of the turbulence is thus highlighted. The effectiveness of the control system in nonlinear field is then evaluated. First, by comparing the bifurcation diagrams for the U and LC system, it is seen the isolation produces an increasing of the limit cycle amplitudes. This (undesired) phenomenon is mainly due to the purely elastic isolation, and it is more evident for very soft springs. On the other hand, it is possible to reduce the oscillation amplitude by adequately designing the nonlinear viscosity coefficient $\zeta_{3}$ of the NLC system. In particular, the value $\zeta_{3}=50$ is found to significantly reduce the amplitudes, with respect both to the $\mathrm{U}$ and LC cases.

The quasi-resonant case is then studied (Fig. 6). First, the influence of detuning on the bifurcation
Fig. 4 Existence domains, in according to the wind parameters $(\bar{U}, \hat{u})$, of the steady solution of the LC system: $\mathbf{a}$ in the resonant case $(\sigma=0)$; $\mathbf{b}$ in a quasi-resonant case ( $\sigma=0.005$ ). In the light and dark gray regions one and two limit cycles exist, respectively. The black lines are the bifurcation loci. The isolation device parameters are $\kappa=8$ and $\zeta_{1}=0.02$

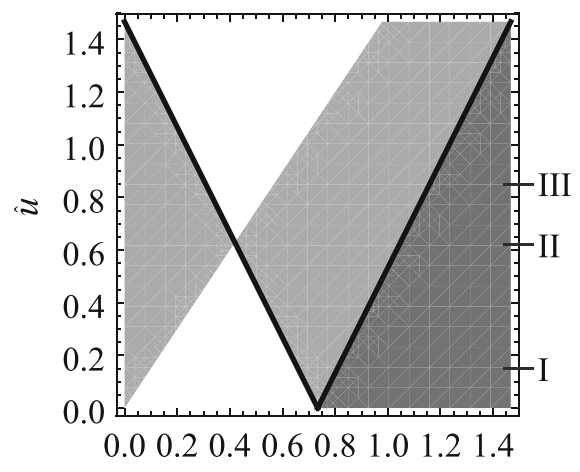

$\bar{U}$

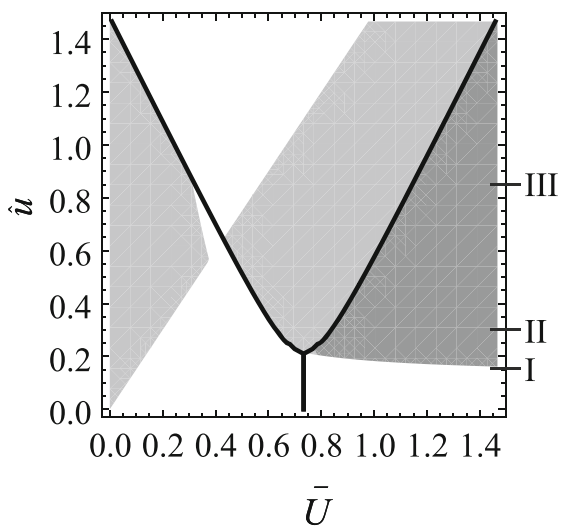

(b) 


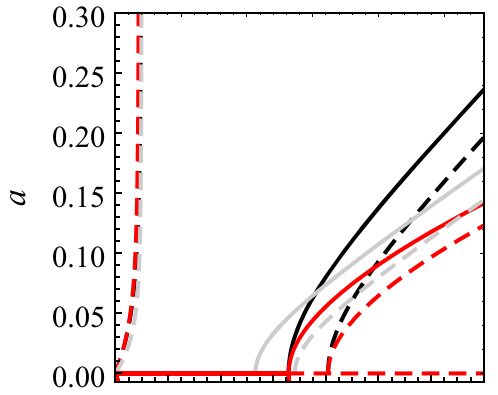

$\begin{array}{lllllllll}0.0 & 0.2 & 0.4 & 0.6 & 0.8 & 1.0 & 1.2 & 1.4\end{array}$ $\bar{U}$

(a)

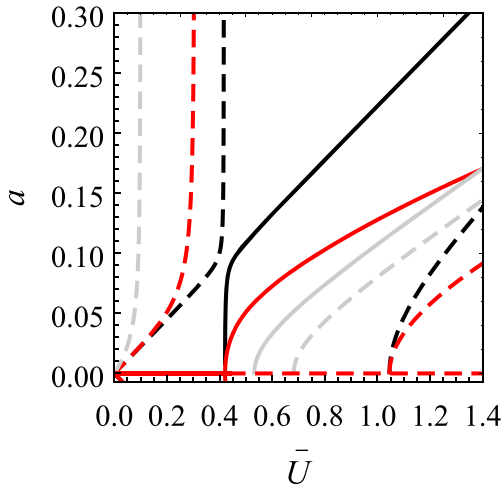

(b)

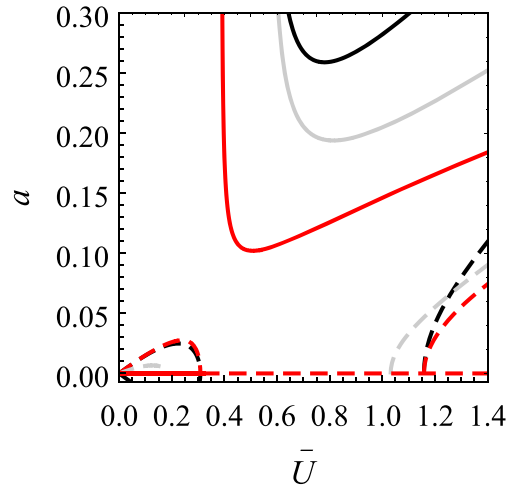

(c)
Fig. 5 Bifurcation diagrams in the resonant case. Steady amplitude of the limit cycle $v s$ the steady wind velocity, for assigned turbulence amplitudes: a $\hat{u}=0.15$ (path I); b $\hat{u}=0.625$ (path II); (c) $\hat{u}=0.85$ (path III). U (gray lines), LC (black lines) and NLC (red lines) system. Unstable and stable solutions are denoted by dashed and solid lines, respectively. The isolation device parameters are $\kappa=8, \zeta_{1}=0.02$ and $\zeta_{3}=50$

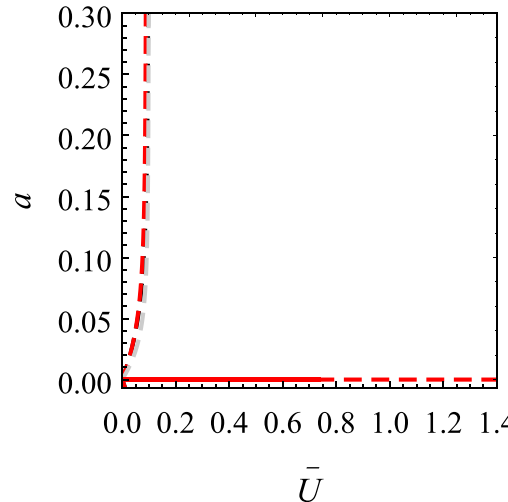

(a)

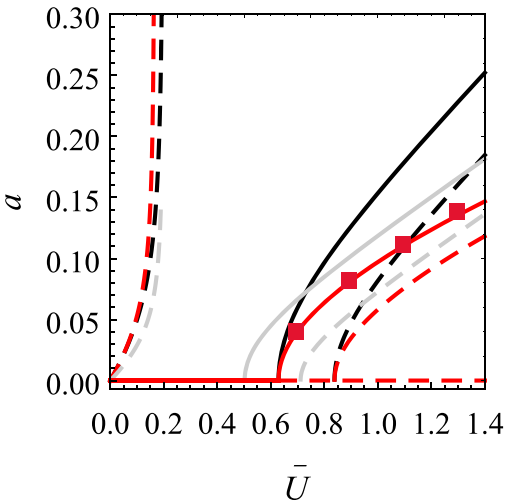

(b)

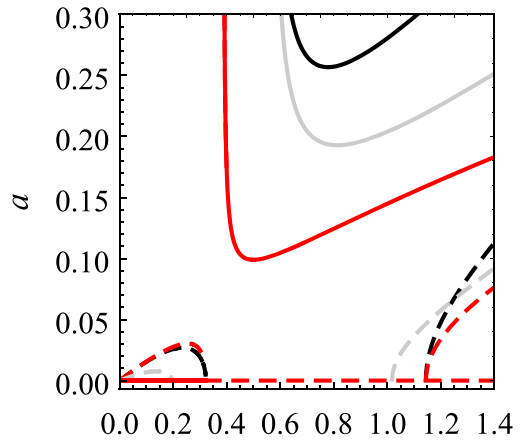

$\bar{U}$

(c)
Fig. 6 Bifurcation diagrams in the quasi-resonant case $\sigma=$ 0.005 . Steady amplitude of the limit cycle $v s$ the steady wind velocity, for assigned turbulence amplitudes: a $\hat{u}=0.15$ (path I); b $\hat{u}=0.3$ (path II); c $\hat{u}=0.85$ (path III). U (gray lines),

paths is highlighted. In particular, the detuning changes the bifurcation scenario with respect to the resonant case, mainly for small values of turbulence amplitude. Indeed, in Fig. 6a, the only (unstable) limit cycle family, bifurcating from $\bar{U}=0$, remains undisturbed. The disappearing of the other two limit cycles is due to the occurrence of a Neimark-Sacker bifurcation (instead of a flip bifurcations) on the trivial path; this aspect will be explored further on. The phenomenology of the paths in Fig. 6b, $c$ is the same of those in Fig. 5a and c for the resonant case. Concerning the isolation influence
LC (black lines) and NLC (red lines) system. Unstable and stable solutions are denoted by dashed and solid lines, respectively. The red markers are the numerical results. The isolation device parameters are $\kappa=8, \zeta_{1}=0.02$ and $\zeta_{3}=50$

on the nonlinear behavior, all the observations made for the resonant case still apply.

It should be noticed that a such post-critical behavior depends on the sign of nonlinear aerodynamic coefficient $A_{3}$ in Eq. (2), according to the shape of the cylinder cross section. Here, it is assumed squared $\left(A_{3}>0\right)$, entailing a supercritical bifurcation (see Fig. 5a and Fig. 6b). When $A_{3}<0$, as in the case of rectangular cross sections with a 2:1 side ratio, a subcritical Hopf bifurcation manifests itself, however, followed by 
Fig. 7 a Trajectories in the state space for different initial conditions (black markers); stable steady solution is denoted by red marker. b Comparison between exact response (gray region) and asymptotic $a(t)$ (black line) time histories. Wind parameters $\bar{U}=0.7$, $\hat{u}=0.3, \sigma=0.005$. The isolation device parameters are $\kappa=8, \zeta_{1}=0.02$ and $\zeta_{3}=50$

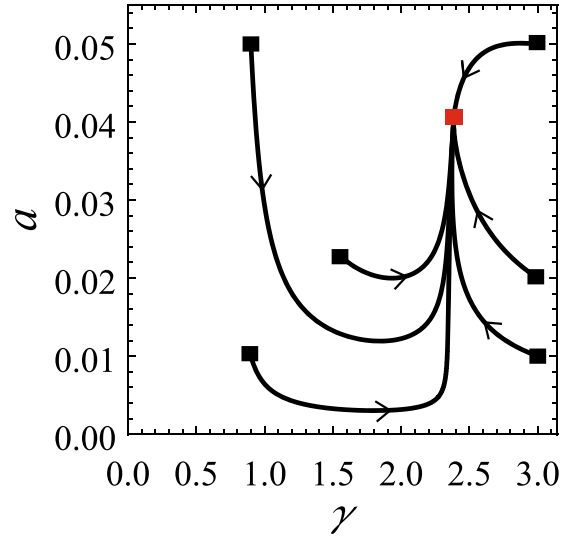

(a)

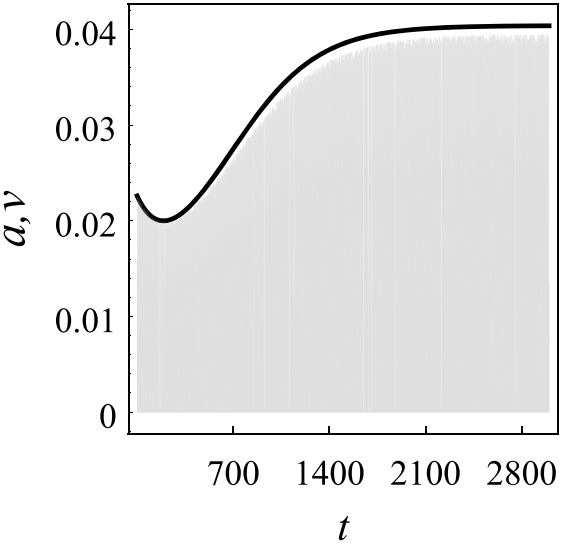

(b) a regain of stability (hard loss of stability phenomenon, as discussed in Ref. [15,22,24]).

To sketch the evolution of the system in the state space during the transient regime, the solutions obtained by direct numerical integrations of the bifurcation Eq. (16), for different the initial conditions, are displayed in Fig. 7a. Here, the $(a, \gamma)$ trajectories are shown for the NLC system and the steady wind velocity $\bar{U}=0.7$, at which only one stable steady solution exists. It is seen that there exists a trajectory, leading to the stable solution, which attracts all the surrounding ones.

Finally, the steady-state and transient asymptotic solutions, are validated against exact finite-difference solutions of the partial differential equations. In particular, for given initial conditions $(a=0.023, \gamma=1.57$ in Fig. 7a), the exact response and the asymptotic $a(t)$ time histories are compared in Fig. 7b. After a transient has been exhausted, the motion stabilizes on a limit cycle of amplitude $a$, depending on the wind parameters. When the limit cycle amplitudes are extracted by the recorded responses, relevant to different wind parameters, the (red) bullets reported in Fig. 6b are found. The agreement between exact and asymptotic solutions is excellent.

\subsection{Torus analysis}

The nonlinear quasi-periodic motions, occurring on tori in the state space, are studied. These solutions are generated by Neimark-Sacker bifurcations, at $\bar{U}=\bar{U}_{c}$. The study is carried out via: (i) finite-difference solution of the partial differential equations Eq. (6), referred ahead as FD solution; (ii) integration of the bifurcation Eq. (12), referred as "numerical solution"; and (iii) asymptotic solution of the slow-slow-flow equation, as described in Sect. 5.2.

The influence of nonlinear viscosity coefficient $\zeta_{3}$ in the NLC system is investigated. A comparison between the asymptotic bifurcation diagrams of the LC and NLC systems is shown in Fig. 8a. At $\bar{U}=\bar{U}_{c}$, a torus bifurcates from the trivial solution. This is represented as an interval $\left[a_{\min }, a_{\mathrm{max}}\right]$ visited by the system during the motion (gray region for the LC system and red region for NLC system). Here, in addition to the quasiperiodic solution, also the periodic solutions are plotted (gray and red dashed lines bifurcating from $\bar{U}=0$ ). It is observed that the addition to the isolation system of a nonlinear damping reduces the amplitude of the tori, but does not change the $a_{\max } / a_{\min }$ ratio, as already observed in Sect. 5.2 (see Eq. 33).

A comparison between FD- (red markers), numerical (black segments) and asymptotic (red region) solutions, for the NLC system, is shown in Fig. 8b. It is observed that: (i) the numerical results of the amplitude modulation equations Eq. (12) are in excellent agreement with the FD results; (ii) the asymptotic solution captures well the maximum amplitude, but it fails in predicting the minimum amplitude; (iii) the $a_{\max } / a_{\min }$ ratio does, indeed, depend on the wind speed, in contrast with the analytical prediction. The inadequacy of the analytical model mainly emerges at high steady wind velocities (far from the critical value), while the accuracy is satisfactory close to the bifurcation. A careful inspection of the order of magnitude of the linear and nonlinear terms in Eqs. (42) and (43) reveals that 
Fig. 8 Bifurcation diagrams: $a$ vs $\bar{U}$ when $\hat{u}=0.15$ and $\sigma=0.005$. a Comparison between asymptotic solutions of the LC (gray regions) and NLC (red region) systems. b Comparison between FD (red markers), numerical (black segments) and asymptotic (red region) periodic solutions in the NLC system. Unstable and stable solutions are denoted by dashed and solid lines, respectively. The isolation device parameters are $\kappa=8, \zeta_{1}=0.02$ and $\zeta_{3}=50$

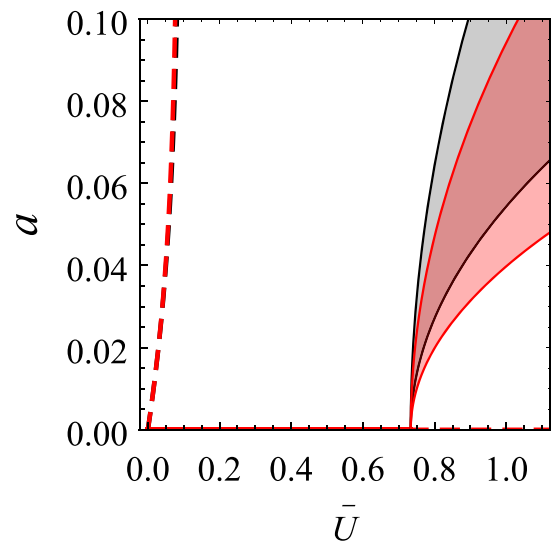

(a)

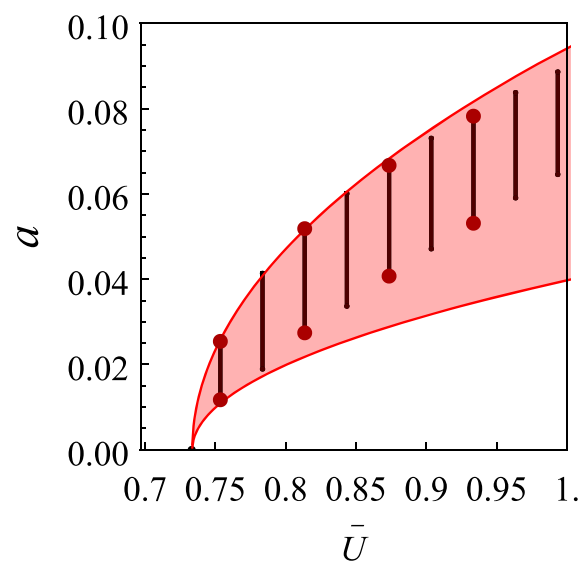

(b) these latter become of the same order of the former for amplitudes $a=0.05$ and incremental wind velocity $\Delta U=0.1$. This denotes a strong nonlinearity of the system, which restricts the validity of the perturbative analysis to a small neighborhood of the critical speed.

For a fixed value of the steady wind velocity (quite far from the bifurcation point), comparisons between numerical and asymptotic solutions for the NLC system are also carried out for: (i) the $X$ and $Y$ time histories, for given initial conditions $X=0.015, Y=0.022$ (Fig. 9a, b); (ii) the periodic orbits in the $(X, Y)$ state space (Fig. 9c); and (iii) the $a$ time histories, for given initial conditions $a=0.054$ (Fig. 9d), together with the FD response (gray region). It is seen that the asymptotic solutions, when compared with the numerical ones, are not very accurate. This is more evident when the periodic solutions are represented in the $(X, Y)$ state space; here they appear as ellipsis, whose axis lengths correspond to the maximum and minimum values of the amplitude $a$. It is observed that the asymptotic and numerical orbits differ in shape and slope. In this regard, it is interesting to observe how this latter evolves with the steady wind velocity (see Fig. 10a, b, referred to asymptotic and numerical solutions, respectively). It turns out that, as the wind velocity increases, the orbits also increase: (a) in isotropic way, i.e., without changing their shape and, therefore, by keeping constant the axis ratio; (b) by passing (to the limit) from an elliptical shape to a circular one, with a consequent decreasing of the axis ratio (that tends to one). Plots are extended up to high motion amplitudes to better show the limit behavior. Indeed, the asymptotic model is not able to capture the evolution of the amplitude ratio. This is due to the fact that the first-order solution (Eq. 25) that just includes the eigenvectors does not account for modification of modal shape with the bifurcation parameter (needed to describe nonlinear normal modes). However, here, the first-order approximation is considered acceptable at realistic low wind speeds.

\section{Conclusions}

The aeroelastic stability of a continuous, homogeneous viscoelastic beam, isolated at the base, under uniform turbulent wind flow, has been studied. The isolation system has been modeled as a nonlinear viscoelastic device; aeroelastic forces computed by the quasi-static theory. The steady component of wind is responsible for a Hopf bifurcation; the turbulent component for parametric excitation. Critical and post-critical behaviors have been analyzed by the multiple-scale method. An efficient control system has been identified, and the mechanical performance of the isolated structure investigated. The asymptotic solutions have validated against nearly exact numerical results, provided by the finite-difference method.

The following main results, concerning linear stability, have been found.

1. The turbulent component modifies the bifurcation scenario, which calls for referring to a threedimensional $(\sigma, \hat{u}, \bar{U})$ parameter space. Here, both flip and Neimark-Sacker bifurcations are likely to occur. Linear instability domains have been determined, for both uncontrolled (U) and linearly controlled (LC) systems. 
Fig. 9 Time histories of: a $X ; \mathbf{b} Y$. c Trajectories in the state space. $\mathbf{d}$ Time histories of $a$ and the FD- response $v$ (gray region). Numerical (black lines) and asymptotic (blue lines) results. Wind parameters $\hat{u}=0.15$, $\sigma=0.005, \Delta U=0.1$. The isolation device parameters $\kappa=8, \zeta_{1}=0.02$ and $\zeta_{3}=50$

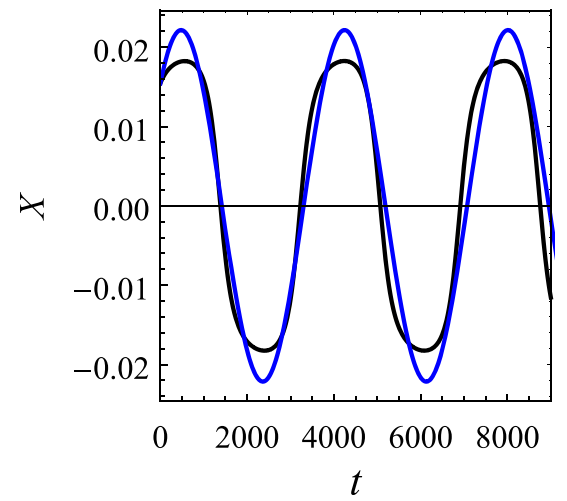

(a)

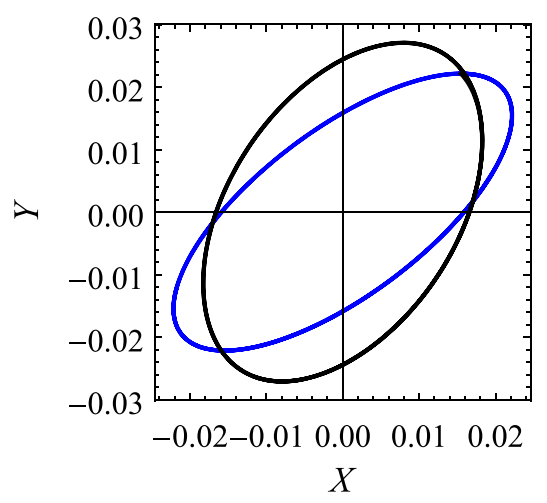

(c)

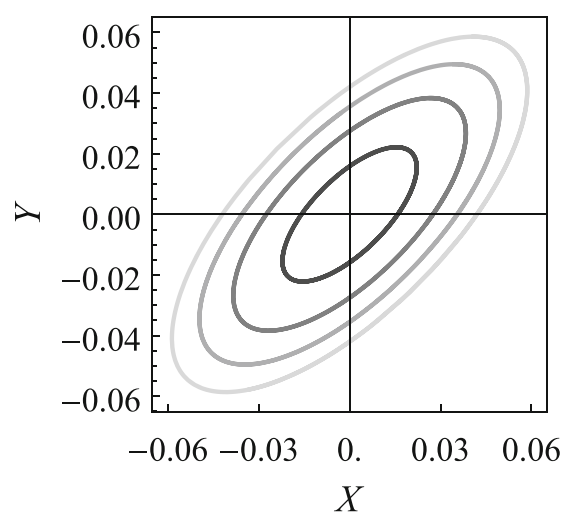

(a)

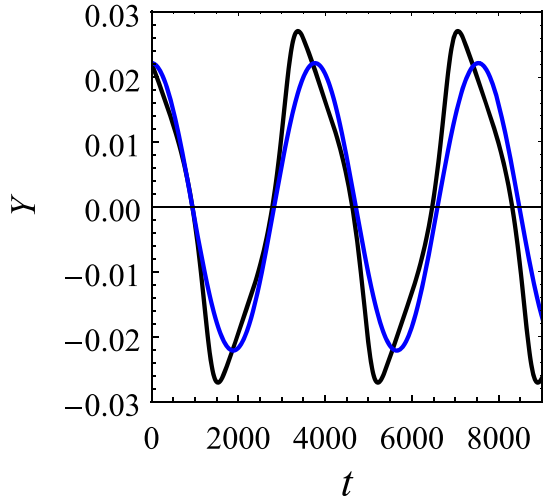

(b)

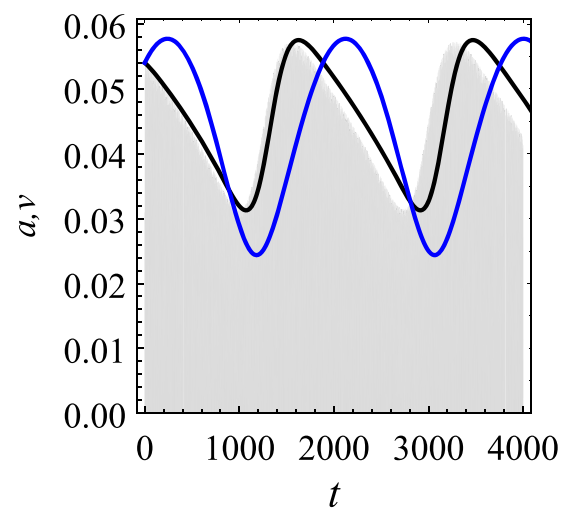

(d)

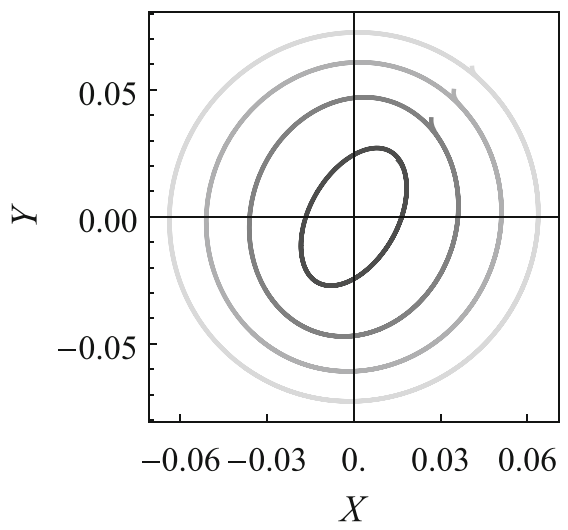

(b)
2. The wind turbulence reduces the galloping velocity via a flip bifurcation.

3. The base isolation entails a translation of the stability boundary along the $\bar{U}$-axis. The purely elastic base isolation, however, produces a detrimental lowering of the critical wind velocity, making essential the addition of a (linear) dashpot. When stiffness and linear damping are opportunely calibrated, the critical galloping velocity is pushed forward.

The following main results, concerning nonlinear behavior, have been found.

1. Limit cycles (periodic motions) and tori (quasiperiodic motions) manifest themselves. Several 
limit cycle branches (just one of which is stable) are generated by flip bifurcations, while tori are generated by Neimark-Sacker bifurcations.

2. Depending on turbulence amplitude and detuning, different bifurcation paths have been found, and some mechanisms of merging and detaching of branches have been analyzed when the bifurcation parameters are varied.

3. A nonlinear dashpot included in the device, when opportunely designed, significantly reduces the oscillation amplitudes, both of the limit cycles and tori.

Finally, an excellent agreement between exact and asymptotic periodic solutions has been found. On the contrary, the asymptotic quasi-periodic solution, when compared with the exact one, captures well the dependence of the maximum amplitude of the motion with the wind speed; however, it is unable to accurately describe the true amplitude interval, especially at high wind velocities.

Funding Open access funding provided by Università degli Studi dell'Aquila within the CRUI-CARE Agreement.

\section{Declarations}

Conflict of Interest The authors declare that they have no conflict of interest.

Open Access This article is licensed under a Creative Commons Attribution 4.0 International License, which permits use, sharing, adaptation, distribution and reproduction in any medium or format, as long as you give appropriate credit to the original author(s) and the source, provide a link to the Creative Commons licence, and indicate if changes were made. The images or other third party material in this article are included in the article's Creative Commons licence, unless indicated otherwise in a credit line to the material. If material is not included in the article's Creative Commons licence and your intended use is not permitted by statutory regulation or exceeds the permitted use, you will need to obtain permission directly from the copyright holder. To view a copy of this licence, visit http://creativecommons.org/licenses/ by/4.0/.

\section{A Bifurcation equation}

The transverse displacement in Eq. (6) is rescaled as $v(s, t) \rightarrow \epsilon v(s, t)$, with $\epsilon \ll 1$ a perturbation parameter. Physical parameters are also rescaled as: $\eta \rightarrow \epsilon \eta, c_{e} \rightarrow \epsilon c_{e}, \zeta_{1} \rightarrow \epsilon \zeta_{1}, \zeta_{3} \rightarrow \epsilon^{-1} \zeta_{3}$, $b_{1} \rightarrow \epsilon b_{1}, b_{3} \rightarrow \epsilon^{-1} b_{3}$. Independent timescales $t_{0}:=t, t_{1}:=\epsilon t, \ldots$, are introduced, so that $\partial_{t}=$ $\partial_{0}+\epsilon \partial_{1}+\ldots, \partial_{t}^{2}=\left(\partial_{0}+\epsilon \partial_{1}+\ldots\right)^{2}$, where $\partial_{j}:=$ $\partial / \partial t_{j}(j=0,1, \ldots)$. Finally, by letting $v(s, t)=$ $v_{0}\left(s, t_{0}, t_{1}, \ldots\right)+\epsilon v_{1}\left(s, t_{0}, t_{1}, \ldots\right)+\ldots$, the following perturbation equations are derived.

Order $\epsilon^{0}$ :

$v_{0}^{\prime \prime \prime \prime}+\partial_{0}^{2} v_{0}=0$

$v_{0 A}^{\prime}=0$

$v_{0 A}^{\prime \prime \prime}+\kappa v_{0 A}=0$

$v_{0 B}^{\prime \prime \prime}=0$

$v_{0 B}^{\prime \prime}=0$

$$
\begin{aligned}
& \text { Order } \epsilon^{1}: \\
& v_{1}^{\prime \prime \prime \prime}+\partial_{0}^{2} v_{1}=-\left(\eta \partial_{0} v_{0}^{\prime \prime \prime \prime}+2 \partial_{0} \partial_{1} v_{0}\right. \\
& +\left(c_{e}+b_{1} \bar{U}\right) \partial_{0} v_{0}+\frac{b_{3}}{\bar{U}}\left(\partial_{0} v_{0}\right)^{3} \\
& \left.+\left(b_{1} \partial_{0} v_{0}-\frac{b_{3}}{\bar{U}^{2}}\left(\partial_{0} v_{0}\right)^{3}\right) \hat{u} \cos \Omega t\right) \\
& v_{1 A}^{\prime}=0 \\
& v_{1 A}^{\prime \prime \prime}+\kappa v_{1 A}=-\left(\eta \partial_{0} v_{0 A}^{\prime \prime \prime}+\zeta_{1} \partial_{0} v_{0 A}+\zeta_{3} \partial_{0} v_{0 A} v_{0 A}^{2}\right) \\
& v_{1 B}^{\prime \prime \prime}=-\eta \partial_{0} v_{0 B}^{\prime \prime \prime} \\
& v_{1 B}^{\prime \prime}=-\eta \partial_{0} v_{0 B}^{\prime \prime}
\end{aligned}
$$

The generating problem Eqs. (34) admit the monomodal solution:

$v_{0}(s, t)=A\left(t_{1}\right) \phi(s) e^{i \omega t_{0}}+$ c.c.

in which $A\left(t_{1}\right)$ is an unknown complex modulating function, c.c. stands for complex conjugate, and $(\omega, \phi(s))$ is the first real eigenpair of the undamped and unloaded beam, constrained at ground by the elastic spring $\kappa$; in particular, $\phi(s)$, normalized according to $\phi(1)=1$, satisfies the following boundary value problem:

$\phi^{\prime \prime \prime \prime}-\omega^{2} \phi=0$

$\phi_{A}^{\prime}=0$

$\phi_{A}^{\prime \prime \prime}+\kappa \phi_{A}=0$

$\phi_{B}^{\prime \prime \prime}=0$

$\phi_{B}^{\prime \prime}=0$

and $\omega$ is a root of the transcendental characteristic equation:

$\frac{\alpha^{3} \tan (\alpha)}{\kappa}+\frac{\alpha^{3} \tanh (\alpha)}{\kappa}=\sec (\alpha) \operatorname{sech}(\alpha)+1$

where $\alpha=\sqrt{\omega}$. 
By substituting the solution Eq. (36) in Eqs. (35), this latter reads:

$$
\begin{aligned}
& v_{1}^{\prime \prime \prime \prime}+\partial_{0}^{2} v_{1}=q_{1}\left(s, t_{0}, t_{1}\right) e^{i \omega t_{0}}+\text { N.R.T. }+ \text { c.c. } \\
& v_{1 A}^{\prime}=0 \\
& v_{1 A}^{\prime \prime \prime}+\kappa v_{1 A}=Q_{1}\left(t_{0}, t_{1}\right) e^{i \omega t_{0}}+\text { N.R.T. }+ \text { c.c. } \\
& v_{1 B}^{\prime \prime \prime}=0 \\
& v_{1 B}^{\prime \prime}=0
\end{aligned}
$$

where N.R.T.stands for non-resonant terms. Finally, the compatibility (or solvability) condition is enforced on the known term:

$$
\int_{0}^{1} q_{1}\left(s, t_{0}, t_{1}\right) \phi(s) d s+Q_{1}\left(t_{0}, t_{1}\right) \phi(0)=0
$$

From Eq. (40), by coming back to the true time $t$, an ordinary differential equation for the amplitude Eq. (10) is obtained.

\section{B Torus asymptotic analysis}

Periodic solutions of the bifurcation Eqs. (12), arising at a Neimark-Sacker bifurcation point, are sought for. The following series expansions are introduced:

$$
\left(\begin{array}{l}
X \\
Y
\end{array}\right)=\left(\begin{array}{c}
X_{0} \\
Y_{0}
\end{array}\right)+\epsilon\left(\begin{array}{c}
X_{1} \\
Y_{1}
\end{array}\right)+\ldots .
$$

and the distinguished bifurcation parameter is split as $\bar{U}=\bar{U}_{c}+\epsilon \Delta U$. A rescaling is performed for the new coefficients: $d_{30} \rightarrow \epsilon^{-1} d_{30}, d_{31} \rightarrow \epsilon^{-1} d_{31}$. By introducing the time scales $t_{0}=t, t_{1}=\epsilon t$, the following perturbation equations are drawn.

Order $\epsilon^{0}$ :

$$
\begin{aligned}
& \partial_{0} X_{0}-\frac{\sigma Y_{0}}{2}+\frac{1}{2} d_{11} \hat{u} X_{0}=0 \\
& \partial_{0} Y_{0}+\frac{\sigma X_{0}}{2}-\frac{1}{2} d_{11} \hat{u} Y_{0}=0
\end{aligned}
$$

Order $\epsilon^{1}$ :

$$
\begin{aligned}
& \partial_{0} X_{1}-\frac{\sigma Y_{1}}{2}+\frac{1}{2} d_{11} \hat{u} X_{1}=-\partial_{1} X_{0}+\Delta U d_{11} X_{0} \\
& +\left(d_{30}+\frac{d_{31}}{\bar{U}_{\mathrm{c}}}+\frac{2 \hat{u} d_{31}}{3 \bar{U}_{c}^{2}}\right) X_{0}^{3} \\
& +\left(d_{30}+\frac{d_{31}}{\bar{U}_{\mathrm{c}}}\right) X_{0} Y_{0}^{2} \\
& \partial_{0} Y_{1}+\frac{\sigma X_{1}}{2}-\frac{1}{2} d_{11} \hat{u} Y_{1}=-\partial_{1} Y_{0}+\Delta U d_{11} Y_{0} \\
& +\left(d_{30}+\frac{d_{31}}{\bar{U}_{\mathrm{c}}}-\frac{2 \hat{u} d_{31}}{3 \bar{U}_{\mathrm{c}}^{2}}\right) Y_{0}^{3}
\end{aligned}
$$

$$
+\left(d_{30}+\frac{d_{31}}{\bar{U}_{\mathrm{c}}}\right) X_{0}^{2} Y_{0}
$$

The generating problem admits the periodic solution:

$$
\left(\begin{array}{c}
X_{0} \\
Y_{0}
\end{array}\right)=R\left(t_{1}\right)\left(\begin{array}{c}
d_{11} \hat{u}-2 i \varpi \\
\sigma
\end{array}\right) e^{i \varpi t_{0}}+\text { c.c. }
$$

where $R\left(t_{1}\right)=\frac{1}{2} r\left(t_{1}\right) e^{i \theta\left(t_{1}\right)}$ is a complex amplitude. With this solution, in order for the $\epsilon^{1}$-equations admit not diverging solutions, resonant terms on the right hand member, of type $\boldsymbol{q} e^{ \pm i \varpi t_{0}}$, must be made orthogonal to the left eigenvectors $\boldsymbol{y}_{ \pm}$, i.e., $\overline{\boldsymbol{y}}_{ \pm}^{T} \boldsymbol{q}=0$. From this condition, by coming back to the true time and unrescaled quantities, the bifurcation equation Eq. (26) is drawn.

\section{References}

1. Novak, M.: Galloping oscillations of prismatic structures. J. Eng. Mech. 98(1), 27-46 (1972)

2. Piccardo, G., Pagnini, L.C., Tubino, F.: Some research perspectives in galloping phenomena: critical conditions and post-critical behavior. Contin. Mech. Thermodyn 27(1-2), 261-285 (2015)

3. Iannelli, A., Marcos, A., Lowenberg, M.: Aeroelastic modeling and stability analysis: a robust approach to the flutter problem. Int. J. Robust Nonlinear Control 28(1), 342-364 (2018)

4. Kim, Taehyoun: Flutter prediction methodology based on dynamic eigen decomposition and frequency-domain stability. J. Fluids Struct. 86, 354-367 (2019)

5. Williamson, C.H.K., Govardhan, R.: Vortex-induced vibrations. Annu. Rev. Fluid Mech. 36, 413-455 (2004)

6. Brika, D., Laneville, A.: Vortex-induced vibrations of a long flexible circular cylinder. J. Fluid Mech. 250, 481-508 (1993)

7. Parkinson, G.V., Wawzonek, M.A.: Some considerations of combined effects of galloping and vortex resonance. J. Wind Eng. Ind. Aerodyn. 8(1-2), 135-143 (1981)

8. Luongo, A.: Zulli, D,: Dynamic instability of inclined cables under combined wind flow and support motion. Nonlinear Dyn. 67(1), 71-87 (2012)

9. Mannini, C., Marra, A.M., Bartoli, G.: Viv-galloping instability of rectangular cylinders: Review and new experiments. J. Wind Eng. Ind. Aerodyn. 132, 109-124 (2014)

10. Sourav, K., Sen, S.: Transition of viv-only motion of a square cylinder to combined viv and galloping at low reynolds numbers. Ocean Eng. 187, (2019)

11. Warminski, Jerzy: Nonlinear dynamics of self-, parametric, and externally excited oscillator with time delay: van der pol versus rayleigh models. Nonlinear Dyn. 99(1), 35-56 (2020)

12. Lu, O.S., To, C.W.S.: Principal resonance of a nonlinear system with two-frequency parametric and self-excitations. Nonlinear Dyn. 2(6), 419-444 (1991) 
13. Szabelski, K., Warmiński, J.: Parametric self-excited nonlinear system vibrations analysis with inertial excitation. Int. J. Non-Linear Mech. 30(2), 179-189 (1995)

14. El-Bassiouny, A.F.: Principal parametric resonances of nonlinear mechanical system with two-frequency and selfexcitations. Mech. Res. Commun. 32(3), 337-350 (2005)

15. Di Nino, S., Luongo, A.: Nonlinear interaction between selfand parametrically excited wind-induced vibrations. Nonlinear Dyn. 1-23, (2020)

16. Abdel-Rohman, Mohamed: Effect of unsteady wind flow on galloping of tall prismatic structures. Nonlinear Dyn. 26(3), 233-254 (2001)

17. Luongo, A., Zulli, D.: Parametric, external and selfexcitation of a tower under turbulent wind flow. J. Sound Vib. 330(13), 3057-3069 (2011)

18. Zulli, Daniele: Luongo, Angelo: Bifurcation and stability of a two-tower system under wind-induced parametric, external and self-excitation. J. Sound Vib. 331(2), 365-383 (2012)

19. Kirrou, Ilham: Mokni, Lahcen, Belhaq, Mohamed: On the quasiperiodic galloping of a wind-excited tower. J. Sound Vib. 332(18), 4059-4066 (2013)

20. Belhaq, Mohamed: Kirrou, Ilham, Mokni, Lahcen: Periodic and quasiperiodic galloping of a wind-excited tower under external excitation. Nonlinear Dyn. 74(3), 849-867 (2013)

21. Zulli, D., Di Egidio, A.: Galloping of internally resonant towers subjected to turbulent wind. Continuum Mech. Thermodyn. 27(4-5), 835-849 (2015)

22. Novak, M.: Aeroelastic galloping of prismatic bodies. J. Eng. Mech. Div 95(1), 115-142 (1969)

23. Luongo, A., D'Annibale, F., Ferretti, M.: Hard loss of stability of ziegler's column with nonlinear damping. Meccanica 51(11), 2647-2663 (2016)

24. Di Nino, S., Luongo, A.: Nonlinear aeroelastic in-plane behavior of suspension bridges under steady wind flow. Appl. Sci. 10(5), 1689 (2020)

25. Bottasso, C.L., Croce, A., Gualdoni, F., Montinari, P.: Load mitigation for wind turbines by a passive aeroelastic device. J. Wind Eng. Ind. Aerodyn. 148, 57-69 (2016)

26. Chen, B., Zhang, Z., Hua, X., Nielsen, S.R., Basu, B.: Enhancement of flutter stability in wind turbines with a new type of passive damper of torsional rotation of blades. J. Wind Eng. Ind. Aerodyn. 173, 171-179 (2018)

27. Vaurigaud, B., Manevitch, L.I., Lamarque, C.-H.: Passive control of aeroelastic instability in a long span bridge model prone to coupled flutter using targeted energy transfer. J. Sound Vib. 330(11), 2580-2595 (2011)

28. Graham, J., Limebeer, M.R., David J.N., Zhao, X.: Aeroelastic control of long-span suspension bridges. J. App. Mech. 78(4) (2011)
29. Casalotti, A., Arena, A., Lacarbonara, W.: Mitigation of post-flutter oscillations in suspension bridges by hysteretic tuned mass dampers. Eng. Struct. 69, 62-71 (2014)

30. Bakis, K.N., Massaro, M., Williams, M.S., Graham, J.M.R.: Passive control of bridge wind-induced instabilities by tuned mass dampers and movable flaps. J. Eng. Mech. 143(9), 04017078 (2017)

31. Winston, C., Feng, M.Q.: Vibration control of super tall buildings subjected to wind loads. Int. J. Non-Linear Mech. 32(4), 657-668 (1997)

32. Lu, Z., Wang, D., Masri, S.F., Lu, X.L.: An experimental study of vibration control of wind-excited high-rise buildings using particle tuned mass dampers. Smart Struct. Syst. 18(1), 93-115 (2016)

33. Di Nino, S., Luongo, A.: Nonlinear aeroelastic behavior of a base-isolated beam under steady wind flow. Int. J. NonLinear Mech. (2019)

34. Ferretti, M., Zulli, D., Luongo, A.: A continuum approach to the nonlinear in-plane galloping of shallow flexible cables. Adv. Math. Phy. 2019, (2019)

35. Novak, M., Davenport, A.G.: Aeroelastic instability of prisms in turbulent flow. J. Eng. Mech. Div. 96(1), 17-39 (1970)

36. Novak, M., Tanaka, H.: Effect of turbulence on galloping instability. J. Eng. Mech. Div. 100(1), 27-47 (1974)

37. Parkinson, G.V., Sullivan, P.P.: Galloping response of towers. J. Wind Eng. Ind. Aerodyn. 4(3-4), 253-260 (1979)

38. Zuo, D., Wu, L., Smith, D.A., Morse, S.M.: Experimental and analytical study of galloping of a slender tower. Eng. Struct. 132, 44-60 (2017)

39. Blevins, D.R.: Flow-induced vibration. Krieger Pub Co, USA (2001)

40. Nayfeh, A.H., Dean T.: Nonlinear oscillations, Mook (1980)

41. Nayfeh, A.H.: Introduction to perturbation techniques. Wiley, New Jersey (2011)

42. Luongo, A.: On the use of the multiple scale method in solving 'difficult' bifurcation problems. Math. Mech. Solids 22(5), 988-1004 (2017)

43. Wijesooriya, K., Mohotti, D., Chauhan, K., Dias-da Costa, D.: Numerical investigation of scale resolved turbulence models (les, eles and ddes) in the assessment of wind effects on supertall structures. J. Build. Eng. 25, 100842 (2019)

44. Robertson, I., Li, L., Sherwin, S.J., Bearman, P.W.: A numerical study of rotational and transverse galloping rectangular bodies. J. Fluids Struct. 17(5), 681-699 (2003)

Publisher's Note Springer Nature remains neutral with regard to jurisdictional claims in published maps and institutional affiliations. 